\title{
Approximate Gradient Direction Metric for Face Authentication
}

\author{
Josef Kittler and Mohammad T. Sadeghi \\ Centre for Vision, Speech and Signal Processing \\ School of Electronics and Physical Sciences \\ University of Surrey, Guildford GU2 7XH, UK \\ \{J.Kittler, M.Sadeghi\}@surrey.ac.uk \\ http://www.ee.surrey.ac.uk/CVSSP/
}

\begin{abstract}
In pattern recognition problems where the decision making is based on a measure of similarity, the choice of an appropriate distance metric significantly influences the performance and speed of the decision making process. We develop a novel metric which is an approximation of the successful Gradient Direction (GD) metric. The proposed metric is evaluated on a face authentication problem using the Banca database. It outperforms the standard benchmark, the normalised correlation. Although it is not as powerful as GD metric, it is ten times faster.
\end{abstract}

\section{Introduction}

In certain pattern recognition applications the training sets are notoriously small. A typical example is biometric person recognition where only a few training data points are available for each individual. An extreme case of the small sample set situation arises in image and video database retrieval, where only a single exemplar is available to define the class of objects of interest.

The usual approach to such problems is to base the decision making on some form of similarity measure, or scoring function, which relates unknown patterns to the query object template. If the degree of similarity exceeds a prespecified threshold, the unknown pattern is accepted to be the same as the query object. Otherwise it is rejected. The similarity concept can also be used in recognition scenarios where the unknown pattern would be associated with that class, the template of which is the most similar to the observed data.

The similarity score is computed in a suitable feature space. Commonly, similarity would be quantised in terms of a distance function, on the grounds that similar patterns will lie physically close to each other. Thus smaller the distance, the greater the similarity of two entities. The role of the feature space in similarity measurement is multifold. First of all the feature space is selected so as to maximise the discriminatory information content of the data projected into the feature space and to remove any redundancy. However, additional benefits sought after from mapping the original pattern data into a feature space is to simplify the similarity measure deployed for decision making. A classical example of this is the use of the Euclidean distance metric in Linear Discriminant Analysis 
(LDA) feature spaces as the within class covariance matrix in the LDA space becomes an identity matrix and such metric becomes theoretically optimal.

Recently we have shown that in some applications, namely personal identity verification based on face biometrics, other scoring functions perform better, even in the LDA space, than the Euclidean distance. One of the examples is the normalised correlation, but the most promising scoring function appears to be the Gradient Direction metric [3]. This has been further generalised in [6]. The main problem with the Gradient Direction metric is its computational complexity.

In this paper we develop an approximation to the Gradient Direction metric which is defined as the difference between the mean (template) of the claimed identity and the local mean of other identities representing the anti-class (impostors). Although not as powerful as the Gradient Direction method, we show that this approximate Gradient Direction metric gives good performance, in comparison with normalised correlation and is significantly simpler to implement than the Gradient Direction metric method.

The paper is organised as follows. In the next section the Gradient Direction metric is reviewed and its approximation is introduced. The experimental set up adopted for the study is detailed in Section 3. The results of experiments are presented in Section 4. A discussion of the results as well as the main conclusions can be found in Section 5 .

\section{Computationally Efficient Gradient Direction Metric}

In a face verification system, a matching scheme measures the similarity or distance of the test sample, $\mathbf{x}$ to the template of the claimed identity, $\boldsymbol{\mu}_{i}$. Note that $\mathbf{x}$ and $\boldsymbol{\mu}_{i}$ are the projection of the test sample and class mean into the feature space respectively. As the simplest solution, a matching score, $s$ for the probe and the $i$ th client mean can be defined as the Euclidean distance between the two vectors, i.e.

$$
s_{E}=\sqrt{\left(\mathbf{x}-\boldsymbol{\mu}_{i}\right)^{T}\left(\mathbf{x}-\boldsymbol{\mu}_{i}\right)}
$$

In [3], it has been demonstrated that a matching score based on Normalised Correlation (NC) is more efficient. The measure is defined as

$$
s_{N}=\frac{\left\|\mathbf{x}^{T} \boldsymbol{\mu}_{i}\right\|}{\sqrt{\mathbf{x}^{T} \mathbf{x} \boldsymbol{\mu}_{i}^{T} \boldsymbol{\mu}_{i}}}
$$

Note that the NC is a similarity measure not a distance metric. The normalised correlation projects the probe vector onto the mean vector of the claimed client identity, emanating from the origin. It effectively uses just one dimensional space onto which the test data is projected. The magnitude of projection is normalised by the length of the mean and probe vectors. The normalised correlation tessellates the probe space into hyper cones or hyper frustums with the axes passing through the origin. It is apparent that the normalised correlation score will be insensitive to probe movements in the radial direction defined by the class mean. 


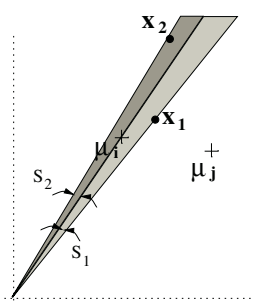

a) Normalised correlation

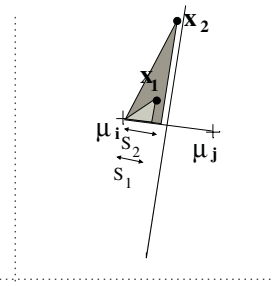

b) Gradient Direction metric

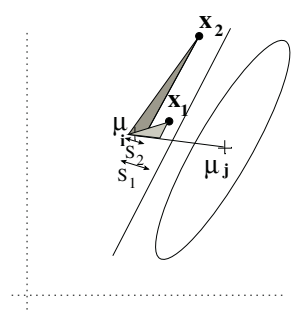

c) Generalised GD metric

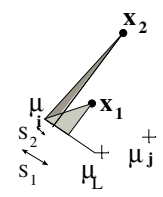

d) Approximate GD metric

Fig. 1. Metrics for matching score definition.

However, the score will drop in value if the probe moves away from this direction angularly. A threshold on the normalised correlation then defines the acceptance region for each client.

Notwithstanding the improvements afforded by the normalised correlation, one can still voice some misgivings. The main drawback of the normalised correlation is that the axes of symmetry of the client acceptance cells are constrained to pass the origin. Inspecting Figure 1-a, this score will not produce the most effective separation of client $i$ from potential imposter $j$.

In [3] an innovate metric called the Gradient Direction metric (GD) has been proposed. In this method the distance between a probe image $\mathbf{x}$ and the $\mathrm{i}$-th client mean vector $\boldsymbol{\mu}_{i}$ is measured along the direction of the gradient of the i-th class aposteriori probability function $P(i \mid \mathbf{x})$. A mixture of Gaussian distributions with the identity covariance matrix has been assumed as the density function of the classes. Note that, this is the same direction along which one should measure distances when using the nearest neighbour decision rule [7]. However the motivation for projecting data on the gradient direction in the case of the nearest neighbour rule is completely different from the above argument for the nearest mean rule used here and we shall therefore not pursue this analogy any further. A conceptually similar direction has been adopted in the nearest feature line method proposed by Li et al. [4].

In [6], we revisited the theory of the Gradient Direction metric and proposed a Generalised Gradient Direction metric. We demonstrated that applying GD metric using either a general covariance matrix derived from the training data or an isotropic covariance matrix with a variance of the order of the variation of the image data in the feature space is even more efficient than the NC scoring function. The proposed optimal matching score has been defined as

$$
s_{O}=\frac{\left\|\left(\mathbf{x}-\boldsymbol{\mu}_{i}\right)^{T} \nabla_{O} P(i \mid \mathbf{x})\right\|}{\left\|\nabla_{O} P(i \mid \mathbf{x})\right\|}
$$

where $\nabla_{O} P(i \mid \mathbf{x})$ refers to the gradient direction. In the generalised form of the GD metric, the optimal direction would be [6]

$$
\nabla_{G} P(i \mid \mathbf{x})=\boldsymbol{\Sigma}^{-1} \sum_{\substack{j=1 \\ j \neq i}}^{m} p(\mathbf{x} \mid j)\left(\boldsymbol{\mu}_{j}-\boldsymbol{\mu}_{i}\right)
$$


where $p(\mathbf{x} \mid j)$ is the $j$-th client measurement distribution. Considering an isotropic structure for the covariance matrix, i.e. $\boldsymbol{\Sigma}=\sigma \mathbf{I}$, equation 4 can be simplified as:

$$
\nabla_{I} P(i \mid \mathbf{x})=\sum_{\substack{j=1 \\ j \neq i}}^{m} p(\mathbf{x} \mid j)\left(\boldsymbol{\mu}_{j}-\boldsymbol{\mu}_{i}\right)
$$

Note that as the length of the gradient vector will have to be normalised anyway the constant on the rhs of the equation can be ignored, however the magnitude of the $\sigma$ will affect the direction through the values of $p(\mathbf{x} \mid j)$. Figure 1-b and 1-c illustrate the geometric differences between these scoring functions. The main drawback of the GD metric is that the method is computationally more expensive than NC function which is a consequence of dependency of the gradient direction on the test data.

Now suppose that the density function $p(\mathbf{x} \mid j)$ of each impostor was very flat (i.e. a large standard deviation). Then the weight factor in (5) defined by the density would effectively be constant and the gradient direction would lose its dependence on the observed probe image. In this case, the optimal direction of projection would be

$$
\nabla P(i \mid \mathbf{x})=\sum_{\substack{j=1 \\ j \neq i}}^{m}\left(\boldsymbol{\mu}_{j}-\boldsymbol{\mu}_{i}\right)
$$

which can be expressed as

$$
\nabla P(i \mid \mathbf{x})=\boldsymbol{\mu}-\boldsymbol{\mu}_{i}
$$

where $\boldsymbol{\mu}$ is the global mean. If the global mean is zero, then the gradient direction is simply defined by the i-th client mean. In the other extreme case, when the standard deviation is very small and the gradient vector is completely dominated by the nearest imposter, the direction of projection will be defined completely by the difference of the i-th client mean and the mean of the nearest imposter.

In practice, none of the above assumptions about the standard deviation is realistic. A reasonable compromise is to consider a small number of the closest neighbours to the claimed identity template to define the Approximate Gradient Direction (AGD) metric as

$$
\begin{aligned}
& \nabla_{A} P(i \mid \mathbf{x}) \cong \sum_{j=1}^{L}\left(\boldsymbol{\mu}_{j}-\boldsymbol{\mu}_{i}\right)=\frac{1}{L} \sum_{j \in \mathcal{N}_{\boldsymbol{\mu}_{i}}^{L}} \boldsymbol{\mu}_{j}-\boldsymbol{\mu}_{i}=\boldsymbol{\mu}_{L}-\boldsymbol{\mu}_{i} \\
& j \neq i \text { and } j \in \mathcal{N}_{\boldsymbol{\mu}_{i}}^{L}
\end{aligned}
$$

where $\mathcal{N}_{\boldsymbol{\mu}_{i}}^{L}$ refers to the set of $L$ such neighbouring templates.

The main advantage of the above formulation is that the direction does not depend on the value of the test sample, $\mathbf{x}$. It will highly speed up the score 
evaluation process as the gradient direction for each client can be calculated in advance. In the next section the Normalised Correlation and Gradient Direction scores will be compared experimentally on the BANCA database [1].

\section{Experimental Design}

In this section the face verification experiments carried out on images of the BANCA database are described. The BANCA database is briefly introduced first. The main specifications of the experimental setup are then presented.

\subsection{BANCA Database}

The BANCA database has been designed in order to test multi-modal identity verification systems deploying different cameras in different scenarios (Controlled, Degraded and Adverse). The database has been recorded in several languages in different countries. Our experiments were performed on the English section of the database. Each section contains 52 subjects (26 males and 26 females). Experiments can be performed on each group separately.

Each subject participated to 12 recording sessions in different conditions and with different cameras. Sessions 1-4 contain data under Controlled conditions while sessions 5-8 and 9-12 contain Degraded and Adverse scenarios respectively. Figure 2 shows a few examples of the face data. Each session contains two recordings per subject, a true client access and an informed imposter attack. For the face image database, 5 frontal face images have been extracted from each video recording, which are supposed to be used as client images and 5 impostor ones. In order to create more independent experiments, images in each session have been divided into two groups of 26 subjects (13 males and 13 females). Thus, considering the subjects' gender, each session can be divided into 4 groups.

In the BANCA protocol, 7 different distinct experimental configurations have been specified, namely, Matched Controlled (MC), Matched Degraded (MD), Matched Adverse (MA), Unmatched Degraded (UD), Unmatched Adverse (UA), Pooled test $(\mathrm{P})$ and Grand test $(\mathrm{G})$. Table 1 describes the usage of the different sessions in each configuration. "T" refers to the client training while " $\mathrm{C}$ " and "I" depict client and impostor test sessions respectively. As we mentioned, 4 groups of data can be considered in each session. The decision function can be trained using only 5 client images per person from the same group and all client images from the other groups. More details about the database and experimental protocols can be found in [1].

\subsection{Experimental Setup}

The original resolution of the image data is $720 \times 576$. The experiments were performed with a relatively low resolution face images, namely $64 \times 49$. The results reported in this article have been obtained by applying a geometric face registration based on manually annotated eyes positions. Histogram equalisation was 


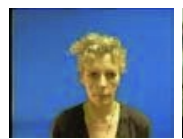

(a)

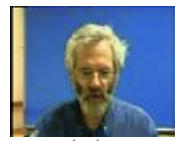

(d)

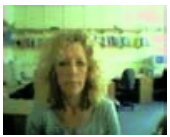

(b)

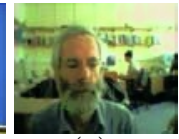

(e)

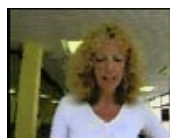

(c)

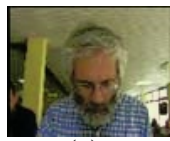

(f)

Fig. 2. Examples of the database images. $a, d$ : Controlled, $b, e$ : Degraded and $c, f:$ Adverse scenarios.

Table 1. The usage of the different sessions in the BANCA experimental protocols.

\begin{tabular}{||c||c|c|c|c|c|c|c|c|c|c|c|c||}
\hline & 1 & 2 & 3 & 4 & 5 & 6 & 7 & 8 & 9 & 10 & 11 & 12 \\
\hline MC & TI & CI & CI & CI & & & & & & & & \\
\hline MD & & & & & TI & CI & CI & CI & & & & \\
\hline MA & & & & & & & & & TI & CI & CI & CI \\
\hline UD & T & & & & I & CI & CI & CI & & & & \\
\hline UA & T & & & & & & & & I & CI & CI & CI \\
\hline P & TI & CI & CI & CI & I & CI & CI & CI & I & CI & CI & CI \\
\hline G & TI & CI & CI & CI & TI & CI & CI & CI & TI & CI & CI & CI \\
\hline
\end{tabular}

used to normalise the registered face photometrically. Linear Discriminant Analysis (LDA) is used for the feature extraction. The XM2VTS database [5] was used for calculating the LDA projection matrix. The thresholds in the decision making system have been determined based on the Equal Error Rate criterion, i.e. where the false rejection rate $(\mathrm{FRR})$ is equal to the false acceptance rate (FAR). The thresholds are set either globally $(G T)$ or using the client specific thresholding $(C S T)$ technique [2]. As we mentioned earlier, in the training sessions of the BANCA database 5 client images per person are available. In the case of global thresholding method, all these images are used for training of the clients template. The other group data is then used to set the threshold. While using the client specific thresholding strategy, only two images are used for the template training and the other three along with the other group data are used to determine the thresholds. Moreover, in order to increase the number of data used for training and to take the errors of the geometric normalisation into account, 24 additional face images per each image are generated by perturbing the location of the eyes position around the annotated positions.

\section{Experimental Results and Discussion}

The performance of different decision making methods based on the Normalised Correlation (NC), the Isotropic Gradient Direction (GD) and the Approximate Gradient Direction (AGD) metrics is experimentally evaluated on the BANCA database using the configurations discussed in the previous section.

Table 2 contains a summary of the results obtained on the test set using the NC and GD methods along with the global and client specific thresholding methods. The values in the table indicate the FAR, FRR and Total Error Rates (TER), i.e. the sum of false rejection and false acceptance rates. In the GD experiments, an isotropic structure $\boldsymbol{\Sigma}=\sigma \mathbf{I}$ with $\sigma$, of the order of the standard deviation of the input data (gray level values) was considered for the impostor distributions. Thus equation 5 is adopted for calculating the gradient directions.

A comparison of the results obtained using the Global and Client Specific thresholding methods indicates that the CST technique is superior in the 
Table 2. ID verification results on BANCA protocols using Normalised Correlation and Gradient Direction methods with Global and Client Specific Thresholding techniques.

\begin{tabular}{|c|c|c|c|c|c|c|c|c|c|c|c|c|}
\hline & \multicolumn{6}{|c|}{ Global Thresholding } & \multicolumn{6}{|c|}{ Client Specific Thresholding } \\
\hline & \multicolumn{3}{|c|}{$\mathrm{NC}$} & \multicolumn{3}{|c|}{ GD } & \multicolumn{3}{|c|}{$\mathrm{NC}$} & \multicolumn{3}{|c|}{ GD } \\
\hline & FAR & FRR & TER & FAR & FRR & TER & FAR & FRR & TER & FAR & FRR & TER \\
\hline $\mathrm{MC}$ & 5.48 & 8.08 & 13.56 & 2.40 & 5.90 & 8.30 & 2.98 & 5.77 & 8.75 & 1.25 & 3.97 & 5.22 \\
\hline MD & 6.35 & 7.18 & 13.53 & 8.36 & 9.61 & 17.97 & 4.14 & 8.20 & 12.34 & 1.25 & 7.05 & 8.30 \\
\hline MA & 8.94 & 9.36 & 18.30 & 7.40 & 7.82 & 15.22 & 5.96 & 10.00 & 15.96 & 1.35 & 6.53 & 7.88 \\
\hline UD & 13.65 & 13.72 & 27.37 & 13.94 & 15.26 & 29.2 & 1.92 & 32.95 & 34.87 & 1.34 & 40.64 & 41.98 \\
\hline $\mathrm{UA}$ & 20.19 & $\overline{21.92}$ & 42.12 & 16.06 & 16.15 & 32.21 & 1.82 & 42.44 & 44.26 & 0.96 & 47.95 & 48.91 \\
\hline $\mathrm{P}$ & 14.01 & 14.23 & 28.24 & 11.57 & 10.64 & 22.21 & 2.18 & 31.45 & 33.63 & 0.99 & 32.48 & 33.47 \\
\hline $\bar{G}$ & 4.84 & 4.61 & 9.45 & 2.18 & 3.33 & 5.51 & 8.20 & 3.33 & 11.54 & 2.02 & 1.58 & 3.60 \\
\hline
\end{tabular}

matched scenario while the GT method gives a better performance on the unmatched protocols. The reason is that, as we mentioned earlier, images in each session have been divided into two groups of 26 subjects. In the GT method, the global threshold of each test group is calculated using the other group of test data. It means that the evaluation and test data have always the same image quality. In the case of the CST technique, we need to have available the client and impostor scores of each client individually. Thus we divided the training images into two subsets, client template training and client scores evaluation images. The impostor scores are then calculated using the other group of the test data (the group which the client does not belong to). It means that in the threshold evaluation of the unmatched experiments, images with different quality are used for calculating the client and impostors scores, while in the test stage all probe images (clients and impostors) have the same quality as the ones which are used for the impostor scores evaluation. Note that $\mathrm{P}$ protocol which is a collection of the MC, UD and UA protocols can mainly be considered as an unmatched protocol while the $\mathrm{G}$ protocol which involves data from different scenarios for both training and test can be seen as a matched protocol.

These results demonstrate clearly that, overall, the best performance is achieved using the Gradient Direction metric.

In the next step, the performance of the Approximate Gradient Direction metric was investigated. The neighbourhood size, $L$ is the most important parameter in the AGD metric. Figure 3 presents the plots of the TER (Total Error Rate) versus the neighbourhood size for the evaluation and test data of different BANCA protocols. Based on the above argument about the thresholding techniques, these results were obtained with the CST method in the case of the matched protocols and GT method in the unmatched cases.

These plots show that by increasing the neighbourhood size the TER first rapidly decreases. Then, for larger values of $L$, the TER remains relatively constant or increases gradually. From these plots, one can also see that the behaviour of TER versus $L$ in the evaluation and test stages is almost consistent. Therefore, the proper neighbourhood size can be found in the evaluation step by looking for the point after which the performance of the system is not significantly improved 

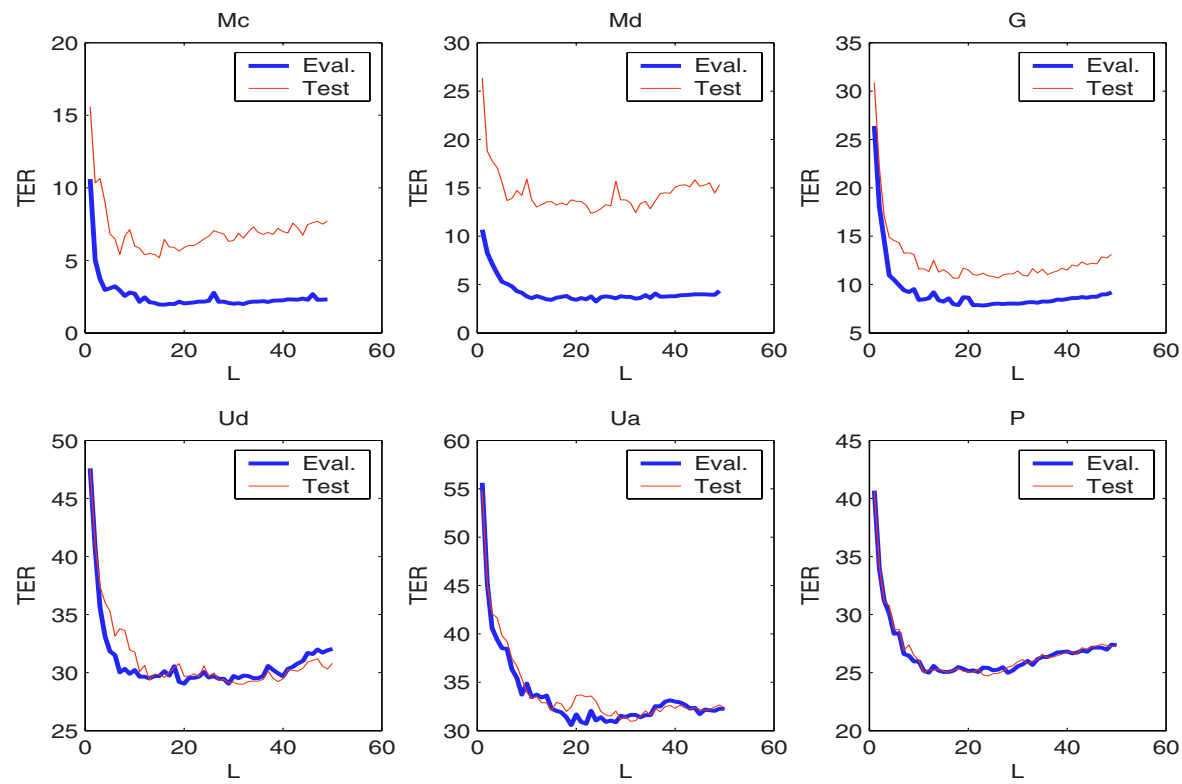

Fig. 3. The performance of the AGD metric versus the neighbourhood size for different BANCA protocols.

by increasing the neighbourhood size. Table 3 contains the results obtained using the proposed AGD method. For the sake of simplicity of comparison, the similar results using the isotropic GD method have also been reported in the table.

These results demonstrate that in the unmatched scenarios, the performance using the AGD method is comparable with the GD technique. However, in the matched scenarios the GD results are better. As mentioned earlier, the main advantage of the AGD method as compared to the GD method is its computational simplicity. The average verification time using the GD metric is around 0.04 CPU units while using the AGD metric it decreases to $0.006 \mathrm{CPU}$ units.

Table 3. Verification results using the AGD and GD methods with global and client specific thresholding methods for unmatched and matched scenarios respectively.

\begin{tabular}{|c||c|c|c||c|c|c|}
\hline \multicolumn{1}{|c||}{} & \multicolumn{3}{c||}{ Approximate GD } & \multicolumn{3}{c|}{ GD } \\
\cline { 2 - 7 } & FAR & FRR & TER & FAR & FRR & TER \\
\hline MC & 2.212 & 3.205 & 5.417 & 1.25 & 3.97 & 5.22 \\
\hline MD & 3.462 & 10.13 & 13.59 & 1.25 & 7.05 & 8.30 \\
\hline MA & 4.904 & 9.103 & 14.01 & 1.35 & 6.53 & 7.88 \\
\hline UD & 15.48 & 14.62 & 30.1 & 13.94 & 15.26 & 29.2 \\
\hline UA & 16.35 & 15.77 & 32.12 & 16.06 & 16.15 & 32.21 \\
\hline P & 12.5 & 12.61 & 25.1 & 11.57 & 10.64 & 22.211 \\
\hline G & 6.923 & 4.274 & 11.2 & 2.02 & 1.58 & 3.60 \\
\hline
\end{tabular}


A comparison of the results obtained using the NC and AGD methods also shows that overall the AGD method is superior, while the computational complexity of the methods (in the test mode) is similar.

\section{Conclusions}

A novel metric which is an approximation of the successful Gradient Direction metric was developed. The performance of face authentication systems in the LDA space using the proposed metric was experimentally compared with the original Gradient Direction (GD) and Normalised Correlation (NC) metrics. The results suggest that the Approximate GD metric outperforms the standard benchmark, the normalised correlation. Although it is not as powerful as GD metric, it is ten times faster.

\section{Acknowledgements}

The financial support from the EU project VAMPIRE is acknowledged.

\section{References}

1. E. Bailly-Baillière, S. Bengio, F. Bimbot, M. Hamouz, J. Kittler, J. Mariéthoz, J. Matas, K. Messer, V. Popovici, F. Porée, B. Ruiz, and J.-P. Thiran. The BANCA database and evaluation protocol. In 4th International Conference on Audio- and Video-Based Biometric Person Authentication, AVBPA, pages 625-638. SpringerVerlag, 2003.

2. K. Jonsson, J. Kittler, Y. Li, and J. Matas. Support vector machines for face authentication. In T. Pridmore and D. Elliman, editors, Proceedings of BMVC'99, pages 543-553, 1999.

3. J. Kittler, Y. P. Li, and J. Matas. On matching scores for LDA-based face verification. In M Mirmehdi and B Thomas, editors, Proceedings of British Machine Vision Conference 2000, pages 42-51, 2000.

4. S.Z. Li and J.W. Lu. Face recognition using the nearest feature line method. IEEE Transactions on Neural Networks, 10(2):439-443, 1999.

5. K. Messer, J. Matas, J. Kittler, J. Luettin, and G. Maitre. XM2VTSDB: The extended M2VTS database. In Second International Conference on Audio and Videobased Biometric Person Authentication, pages 72-77, March 1999.

6. M. Sadeghi and J. Kittler. Decision making in the LDA space: Generalised gradient direction metric. In the 6th International Conference on Automatic Face and Gesture Recognition, Seoul, Korea, May 2004.

7. R.D. Short and K. Fukunaga. The optimal distance measure for nearest neighbour classification. IEEE Transactions on Information Theory, 27(5):622-627, 1981. 